ORIGINAL ARTICLE

\title{
External quality assessment schemes raise standards: evidence from the UKNEQAS parasitology subschemes
}

\author{
M M Kettelhut, P L Chiodini, H Edwards, A Moody
}

J Clin Pathol 2003;56:927-932

See end of article for authors' affiliations ....................

Correspondence to: Professor P L Chiodini, Department of Clinical Parasitology, Hospital for Tropical Diseases, Mortimer Market, Capper Street, London WCIE 6AU, UK: peter.chiodini@uclh.org

Accepted for publication 11 May 2003
Background: The burden of parasitic disease imported into the temperate zone is increasing, and in the tropics remains very high. Thus, high quality diagnostic parasitology services are needed, but to implement clinical governance a measure of quality of service is required.

Aim: To examine performance in the United Kingdom National External Quality Assessment Scheme for Parasitology for evidence of improved standards in parasite diagnosis in clinical specimens.

Methods: Analysis of performance was made for the period 1986 to 2001, to look for trends in performance scores.

Results: An overall rise in performance in faecal and blood parasitology schemes was found from 1986 to 2001. This was seen particularly in the identification of ova, cysts, and larvae in the faecal scheme, the detection of Plasmodium ovale and Plasmodium vivax in the blood scheme, and also in the correct identification of non-malarial blood parasites. Despite this improvement, there are still problems. In the faecal scheme, participants still experience difficulty in recognising small protozoan cysts, differentiating vegetable matter from cysts, and detecting ova and cysts when more than one species is present. In the blood scheme, participants have problems in identifying mixed malarial infections, distinguishing between $P$ ovale and $P$ vivax, and estimating the percentage parasitaemia. The reasons underlying these problems have been identified via the educational part of the scheme, and have been dealt with by distributing teaching sheets and undertaking practical sessions.

Conclusions: UK NEQAS for Parasitology has helped to raise the standard of diagnostic parasitology in the UK.
"The ability to measure the quality of service is essential for successful implementation of a culture that supports clinical governance." ${ }^{\prime 1}$

$\mathrm{F}$ ollowing the discontinuation in 1972 of the Institute of Biomedical Sciences (formerly known as the Institute of Medical Laboratory Sciences) examination in parasitology, and the retirement of former armed forces Biomedical Scientists (formerly known as Medical Laboratory Scientific Officers), laboratory expertise in parasitology was less widespread in the UK and problems were encountered in the identification of parasites in clinical specimens, yet the burden of imported parasitic disease in the UK was increasing. Thus, in the early 1980s, a pilot Parasitology External Quality Assessment (EQA) survey was undertaken by Ridley (unpublished), which showed poor performance among UK diagnostic laboratories. In 1986, the Hospital for Tropical Diseases (under the wing of the Quality Assurance Laboratory of the Public Health Laboratory Service) was asked to develop a Parasitology EQA scheme. ${ }^{2}$ This was organised for faecal and blood parasites and followed by a United Kingdom Quality Assessment Scheme (UKNEQAS) associated teaching scheme in 1993.

Snell $^{3}$ has described the benefits of participation in an EQA scheme for laboratories: (1) participating laboratories are able to assess whether their results are comparable with those of other laboratories; (2) EQA can provide a valuable educational stimulus to laboratory staff; (3) it provides credibility to the participating laboratory by providing evidence that the participating laboratory has a responsible attitude towards quality issues (evidence of participation is required by some accrediting agencies); (4) EQA provides an insight into national performance levels; and (5) EQA improves national performance levels.

The UKNEQAS in Parasitology is designed to: (1) improve the diagnosis of parasitic disease by examination of clinical material from patients with parasitic infections; and (2) provide teaching material illustrating unusual or uncommon parasites and targeting areas where a particularly poor performance was noted.

In the faecal parasitology scheme, eight distributions, each containing two specimens, are currently despatched to 618 participants ( 301 from the UK and 317 from overseas) each year. At the start of the scheme in 1986, 452 laboratories (356 from the UK and 96 from overseas) participated.

\section{"External quality assessment can provide a valuable educational stimulus to laboratory staff"}

In the blood parasitology scheme, eight distributions, each containing one specimen, are currently despatched to 285 participants (91 from the UK and 194 from overseas) each year. At the start of the scheme, 252 laboratories (134 from the UK and 118 from overseas) participated.

Currently, $86 \%$ of UK microbiology laboratories who subscribe to the UKNEQAS General Bacteriology Scheme also subscribe to the faecal parasitology scheme and $15 \%$ subscribe to the blood scheme. The low figure for microbiology participation in the blood scheme compared with the

Abbreviations: EQA, external quality assessment; UKNEQAS, United Kingdom External National Quality Assessment Scheme 


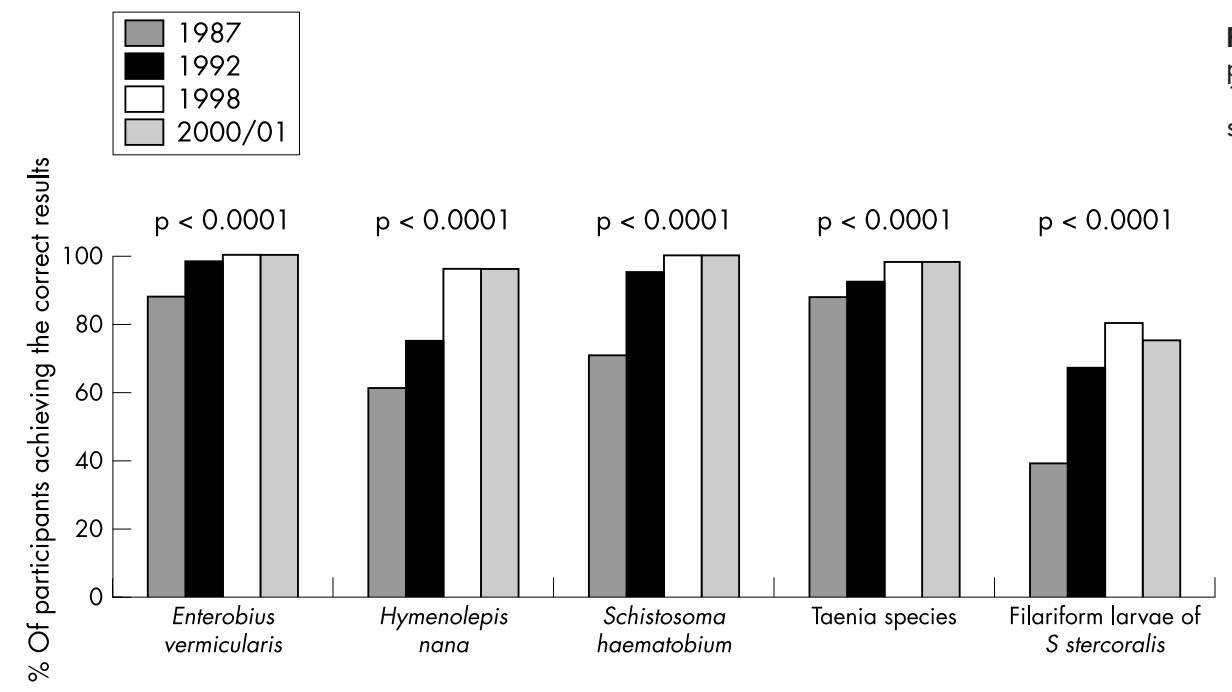

Figure 1 Comparison of participants' performance in the years 1987, 1992 1998 , and $2000 / 1$ in examining faecal specimens for helminths.

faecal scheme is because, in the UK, most blood films for parasites are examined in haematology laboratories.

\section{MATERIALS AND METHODS}

In the faecal parasitology scheme, the material distributed contains a wide range of parasites present in formalised faecal suspensions, fixed or stained faecal smears, formalised urine suspensions, and formalised cyst fluids. Most specimens are obtained from overseas specimen collection trips, but some are sourced from the department of clinical parasitology, Hospital for Tropical Diseases, UK, or from specialist institutes.

In the blood parasitology scheme, the material distributed contains a wide selection of blood and tissue parasites present in thick and thin blood films and tissue dabs. Most of these specimens are obtained from the department of clinical parasitology, but they may also be obtained from specialist institutes.

An important component of UKNEQAS for Parasitology is education and the following components provide educational support for participants: when an unusual parasite is distributed or a particularly poor performance is noted, a teaching sheet that outlines the life cycle, the clinical presentation, and the laboratory diagnosis of the parasite in question is issued. This allows the participant to take individual action to investigate and remedy any defects revealed in their current methodology.

UKNEQAS may also act as a medium for introducing clinical laboratories to unusual or "new" parasites, which they otherwise would not see. Such new parasites were Cyclospora cayetanensis in 1993 and more recently spores of microsporidia. Future distributions will include acanthamoeba species.

New techniques have also been introduced to laboratory practice via the scheme-for example, the modified version of the trichrome stain for the detection of microsporidial spores in faecal specimens from immunocompromised patients with diarrhoea.

In April 1993, a NEQAS Parasitology associated teaching scheme for blood and faecal parasites was begun for participants in the parasitology subschemes. This extended teaching programme offers "hands on" practical experience in parasitology techniques by running regional courses throughout the UK and Eire. Through this teaching programme, many problems in identifying parasites have been highlighted. From April 1994, the teaching scheme was offered to participants in the UKNEQAS General Haematology scheme.

In this study, the performance data for faecal and nonintestinal parasites and blood (including tissue) parasites was taken from specimens distributed between 1986 and 2001 from UK laboratories. These data were analysed by organism and year to look for trends in performance. A $\chi^{2}$ test was performed to establish whether the trends observed were significant.

\section{RESULTS}

The results in figs 1,2 , and 3 demonstrate a significant overall improvement in detection of faecal parasites for all the organisms shown when the performance for 1986 is compared with that for 2001. Furthermore, when overall performance for those periods is compared, there was a significant increase in the proportion of UK participants achieving scores of $80-100 \%$ (fig 4 ). If analysis is restricted to those UK participants who have subscribed since the start of the scheme, in case early poor performers withdrew from UKNEQAS for Parasitology, which would have biased the
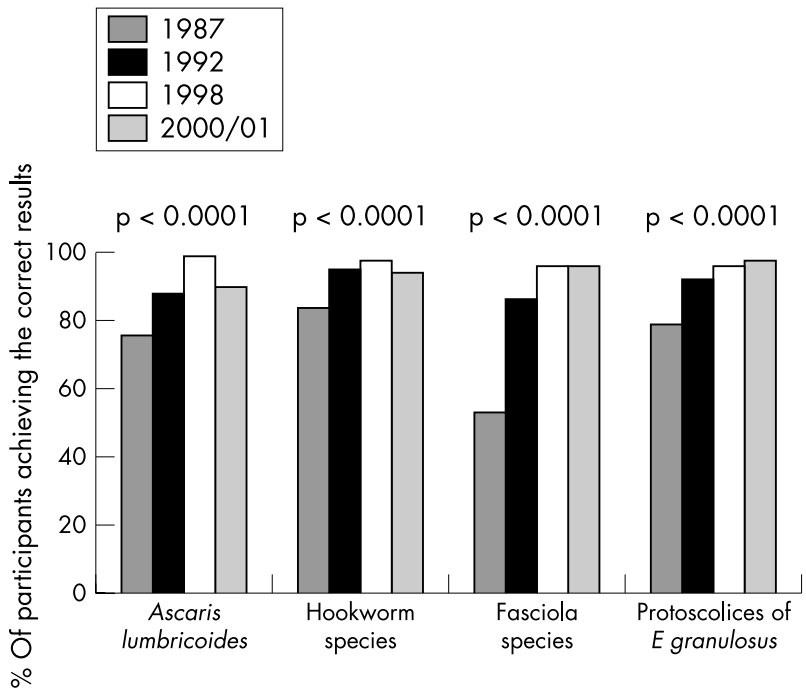

Figure 2 Figure 1 Comparison of participants' performance in the years 1987, 1992, 1998, and 2000/1 in examining faecal specimens for helminths. 


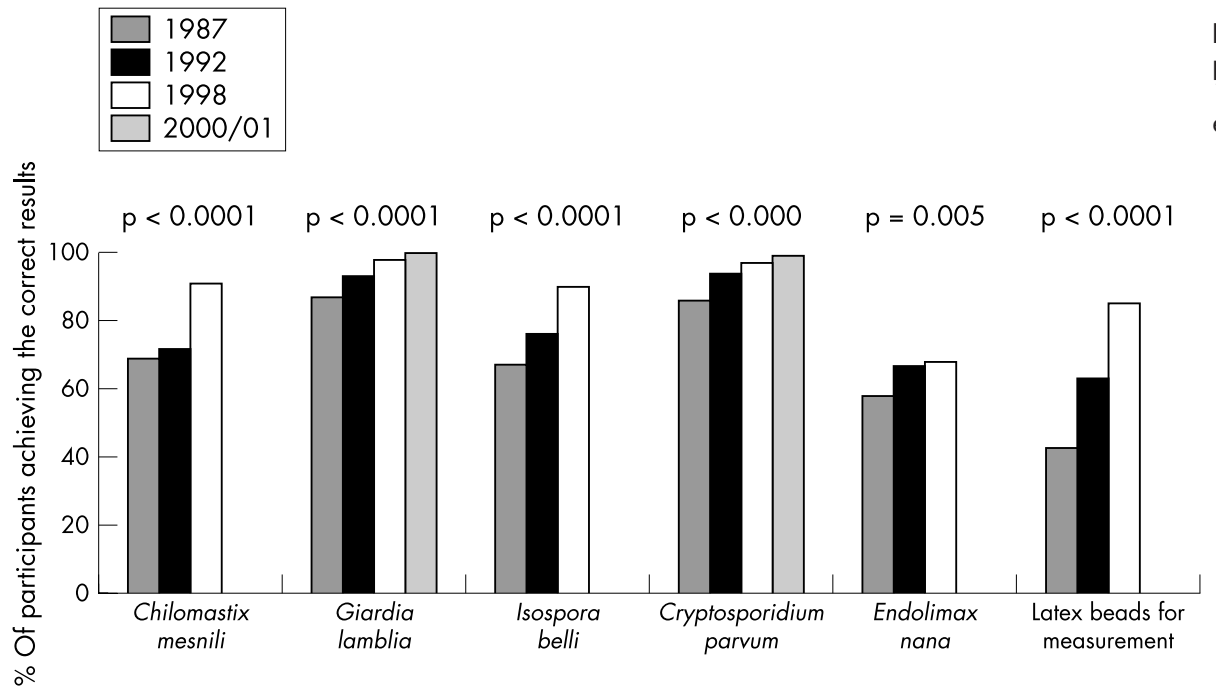

Figure 3 Figure 1 Comparison of participants' performance in the years $1987,1992,1998$, and $2000 / 1$ in examining faecal parasites for cysts.

sample, there was still a significant increase in the proportion achieving scores of $80-100 \%$ (fig 5 ).

Figures 6 and 7 show an overall improvement in the detection of all blood parasites. As in the faecal scheme, there has been a significant increase in the total number of UK participants achieving scores of $80-100 \%$ (fig 8 ), and a significant increase in the number of UK participants who have subscribed since the start of the scheme achieving scores of $80-100 \%$ (fig 9).

Although there has been a very encouraging rise in standards for the detection of both faecal and blood parasites, there are still problems that must be dealt with, as follows.

\section{Faecal parasitology scheme}

Participants have difficulty in recognising and identifying small protozoan cysts measuring less than $10 \mu \mathrm{m}$. For example, when cysts of Endolimax nana or Chilomastix mesnili were distributed, either alone or with another parasite, 25$37 \%$ of laboratories failed to report them.

Confusion of vegetable matter with parasite material. Some plant cells, pollen grains, and yeasts can resemble parasites and participants frequently confuse them with ova and cysts. For example, in two separate specimens containing pollen grains, but no parasites, $8 \%$ and $14 \%$, respectively, of participants reported the presence of various ova and cysts.

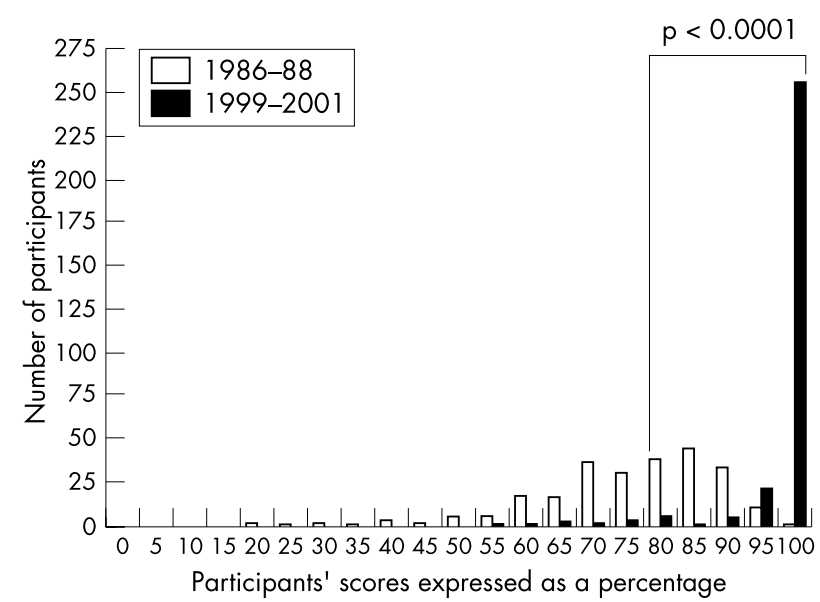

Figure 4 National External Quality Assessment Scheme for Faecal Parasitology: all UK participants.
Failure to detect all species when ova and cysts from more than one parasite are present is still observed. In specimens containing two or three parasites, only $30-60 \%$ of participants correctly identified all parasites present, compared with $70-98 \%$ if there is only a single species of parasite in the sample.

Some participants still fail to report the stage of helminth larvae seen; $25-61 \%$ of them failed to report the stage of Strongyloides stercoralis present (fig 1). Points are deducted for this omission because failing to report the stage has implications for clinical management, because numerous filariform larvae in a freshly collected stool specimen would point towards the presence of the strongyloides hyperinfestation syndrome.

\section{Blood parasitology scheme}

Estimation of the percentage parasitaemia of Plasmodium falciparum continues to cause problems. Many participants failed to calculate the parasitaemia at all, and for those who did, $13-39 \%$ of them failed to do so correctly. Where it is estimated wrongly, the tendency is to overestimate the percentage.

Performance on specimens where more than one plasmodium species is present remains poor. For example, only 13$27 \%$ of reports showed correct identification of both malaria species in mixed infections, compared with 76-92\% correct for single species distributions.

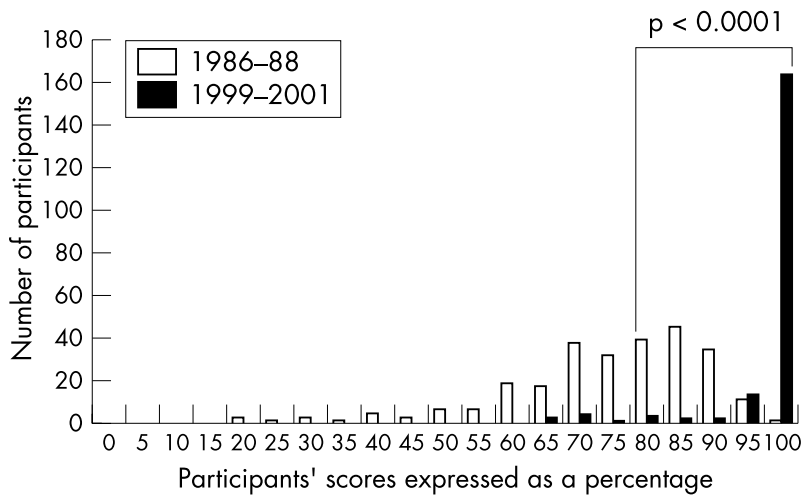

Figure 5 UK National External Quality Assessment Scheme for Faecal Parasitology: participants subscribing since the start of the scheme. 


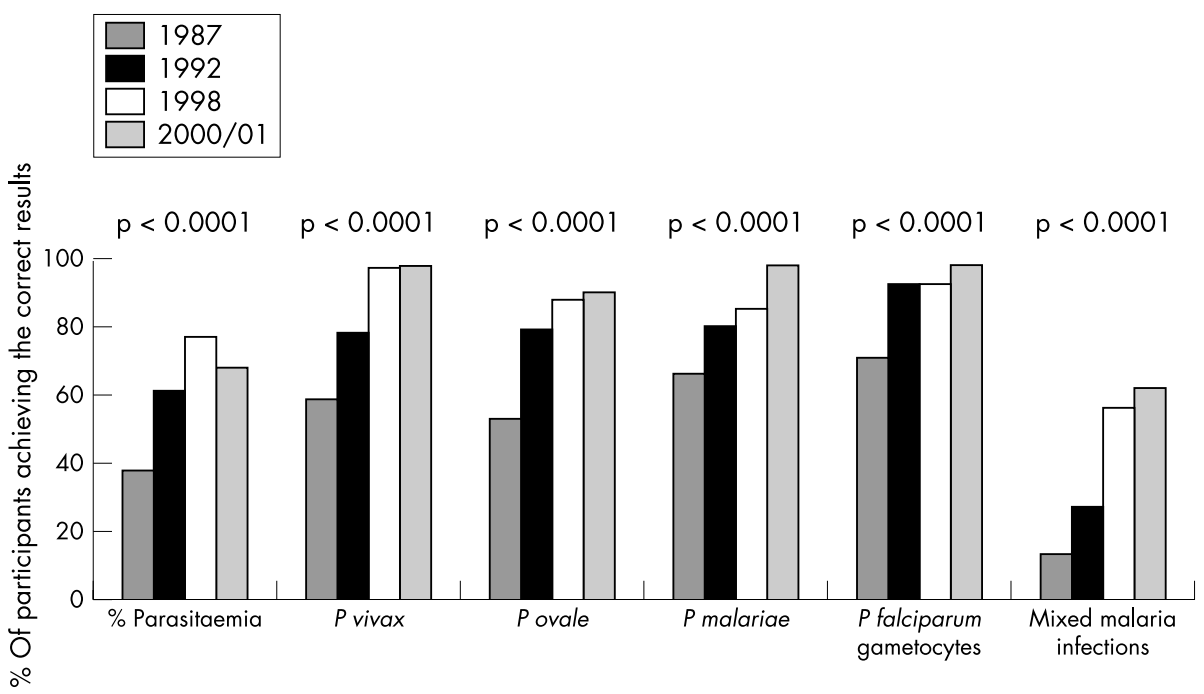

Figure 6 Comparison of participants' performance in the years 1987, 1992, 1998 , and $2000 / 1$ in examining blood films for malaria parasites.

Given the morphological similarity of Plasmodium vivax and Plasmodium ovale, some confusion between them might be expected, and that was indeed the case, with $14-42 \%$ of participants mistaking one of these malaria species for the other.

Some scheme members confuse Howell-Jolly bodies, fibrin strands, and artefacts such as stain granules with blood parasites. Depending on the distribution, $8-15 \%$ of participants reported a variety of blood parasites in negative blood films.

The results of the UKNEQAS Parasitology schemes also demonstrate several successes and distributions in which participants scored particularly well were:

\section{Faecal parasitology scheme}

- Specimens containing ova of a single species of helminth, where $90-96 \%$ of participants achieved the correct results.

- Non faecal specimens, such as urine or cyst fluid, where 96-100\% of participants achieved the correct results.

- Specimens containing parasites that are encountered frequently in routine practice, such as Cryptosporidium parvum or Giardia lamblia, where almost $100 \%$ of participants achieved the correct results.

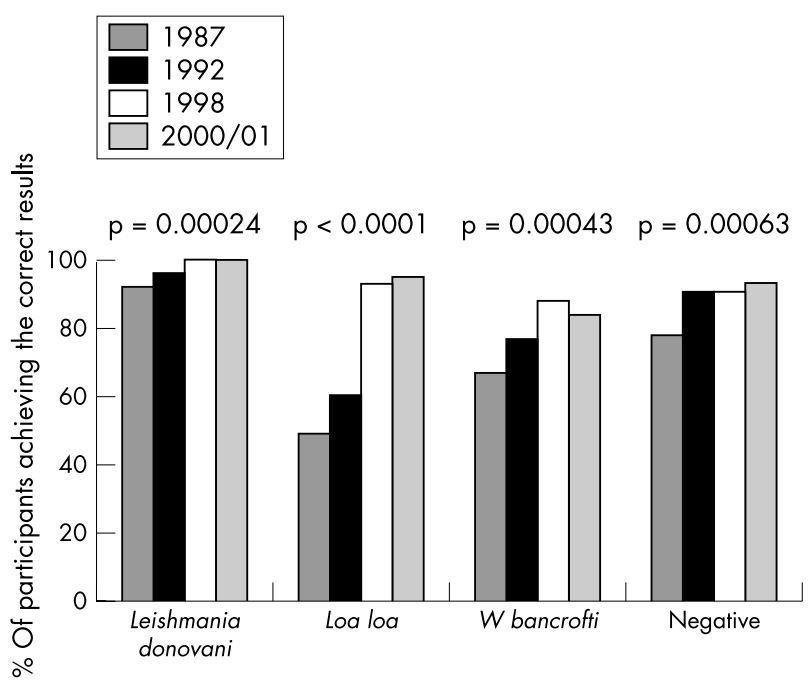

Figure 7 Comparison of participants' performance in the years 1987, 1992,1998 , and $2000 / 1$ in the examination of blood films.

\section{Blood parasitology scheme}

- Blood films that contained familiar malaria parasites, such as the characteristic gametocytes of $P$ falciparum, where approximately $93 \%$ of reports gave the correct results.

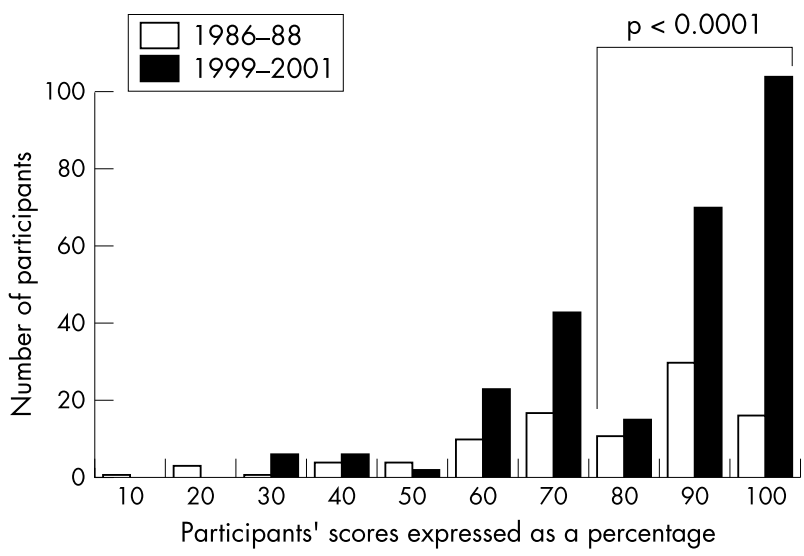

Figure 8 National External Quality Assessment Scheme for Blood Parasitology: all UK participants.

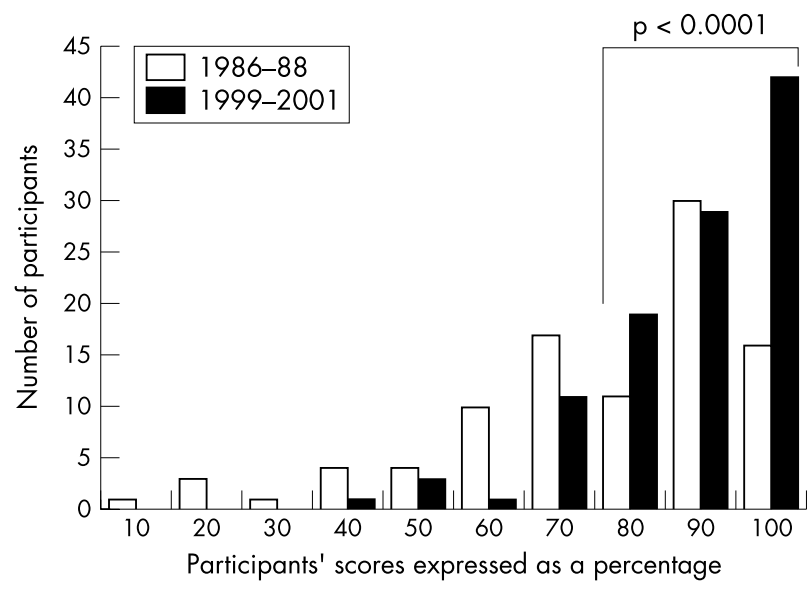

Figure 9 UK National External Quality Assessment Scheme for Blood Parasitology: participants subscribing since the start of the scheme. 


\section{Take home message}

- The United Kingdom Quality Assessment Scheme for Parasitology appears to have had a direct effect on raising the awareness of parasitic infection in UK laboratory practice, and in ensuring more uniform application of standard diagnostic methods

- It has done this by highlighting problem areas, and providing focused teaching

- Blood films that contained non-malaria parasites-that is, microfilariae or trypanosoma species-where approximately $93 \%$ were reported correctly.

- Specimens that contained tissue parasites, such as Leishmania spp, in which $95-100 \%$ of participants achieved the correct results.

\section{DISCUSSION}

An analysis of specimens distributed to UK laboratories between the start of the scheme in 1986 and 2001 showed a significant improvement in performance in both faecal and blood parasitology schemes for all of the parasites examined (figs 1-9).

Why are participants achieving better results? Possible reasons suggested are: (1) the teaching sheets that are sent out with the UKNEQAS reports; (2) the UKNEQAS Parasitology associated teaching scheme; (3) laboratory closures or amalgamations, such that poor performers are eliminated; or (4) a mixture of the above.

We think that the education and training provided by NEQAS, especially via its teaching arm, has played an important part. Data shown in figs 5 and 9 exclude the possibility of laboratory closures accounting for improvement because they show the performance trends for laboratories that have been enrolled throughout the lifetime of the UKNEQAS Parasitology schemes. Furthermore, according to the scheme records, those laboratories that discontinued their subscriptions were not shown to be poor performers.

However, problems with parasite identification still exist. In the faecal parasitology scheme, participants still have problems with identifying small cysts, confusing vegetable matter with cysts, identifying ova and cysts when there is more than one species present, and failing to specify the stage of the parasite.

Since the start of the NEQAS associated teaching programme in April 1993, many reasons for these difficulties became apparent as the NEQAS staff met increasing numbers of participants face to face on training days.

Some laboratories in Britain are not called upon to examine stools routinely for parasites, because they do not serve a population that would commonly harbour parasites. In some weeks, the only parasite positive stools that these laboratories might examine are those sent from the quality assessment scheme.

Some laboratories do not use a recognised concentration technique, or do not concentrate stool specimens at all. This leads to a poor recovery, yielding low numbers of parasites, especially cysts, which can be missed completely because of masking by excess faecal matter.

Some laboratories do not use iodine as a primary stain on wet faecal preparations. This contributes to their inability to distinguish between vegetable matter and cysts. Iodine highlights the internal structure of cysts, thus facilitating identification.
Some laboratories do not possess an eyepiece graticule. The inability to measure correctly or failure to measure makes precise identification much more difficult and could lead to wrong speciation.

Many laboratories do not examine a sufficient quantity of faecal material, so that parasites which are present in small numbers may be missed completely.

In the blood parasitology scheme, participants have problems with calculating the parasitaemia of $P$ falciparum, confusing $P$ vivax and $P$ ovale, identifying plasmodium species when there is more than one species present, and confusing blood artefacts with blood parasites.

Since the start of the NEQAS Parasitology associated teaching programme in April 1993 reasons for these difficulties have become apparent.

\section{"The persisting problem areas in both the schemes are constantly being dealt with in the teaching sheets"}

Some laboratories in Britain receive very few requests for the examination of blood parasites. In some weeks the only malaria positive blood films which these laboratories might examine are those from the quality assessment scheme, resulting in a lack of confidence in identifying blood parasites, especially in the speciation of malaria.

Some participants overestimate the percentage parasitaemia of $P$ falciparum as a result of counting the number of parasites for each 100 red blood cells, as opposed to the percentage number of parasitised red blood cells, and some fail to calculate the parasitaemia correctly because of mathematical errors.

Some participants do not spend sufficient time in examining a blood film and therefore miss characteristic features of blood parasites. This is particularly noticeable when differentiating between $P$ ovale and $P$ vivax, or when examining films with mixed malaria infections.

The persisting problem areas in both the schemes are constantly being dealt with in the teaching sheets, which are distributed to participants and via the UKNEQAS associated teaching scheme.

Regarding overall performance, we are aware that there is a risk that EQA data might represent the best possible work of a laboratory, rather than its day to day standard, because participants know when they are examining an EQA specimen. However, Milne et $\mathrm{al}_{,}{ }^{4}$ in a study conducted in 1991 on the accuracy of routine laboratory diagnosis of malaria in the UK, found a similar proficiency in diagnosis among laboratories submitting slides for reference confirmation and laboratories reporting on NEQAS specimens, suggesting that the results we report here are representative of the general situation. UKNEQAS for Microbiology ${ }^{5}$ also noted that performance improved over the years, albeit slowly and, like us, documented repetition of errors by a minority of participants. However, the overall level of performance in the microbiology scheme is higher and their participants started from a higher baseline-for example, in 1992, 97\% of reports in that scheme received for each strain/antimicrobial combination were correct.

\section{CONCLUSIONS}

We believe that UKNEQAS for Parasitology has been of direct benefit in raising awareness of parasitic infection in UK laboratory practice, and in ensuring more uniform application of standard diagnostic methods. By highlighting problem areas, and providing focused teaching, it has helped to raise the standard of diagnostic parasitology in the UK. 


\section{ACKNOWLEDGEMENTS}

We acknowledge the help of Ms F Warburton from the Department of Statistics at the Central Public Health Laboratory, Colindale, London, UK.

\section{Authors' affiliations}

M M Kettelhut, P L Chiodini, H Edwards, A Moody, UKNEQAS

Parasitology, Department of Clinical Parasitology, Hospital for Tropical Diseases, Mortimer Market, Capper Street, London WC1E 6AU, UK

\section{REFERENCES}

1 Halligan A, Donaldson L. Implementing clinical governance: turning vision into reality. BMJ 2001;322:1413-17.

2 Hawthorne M, Chiodini PL, Snell JJS, et al. Parasitology Quality Assessment: United Kingdom National Scheme. J Clin Pathol 1992;45:968-74.

3 Snell JJS. External quality assessment. In: Snell JJS, Brown DFJ, Roberts C, eds. Quality assurance. Principles and practice in the microbiology Laboratory. London: Public Health Laboratory Service, 1999:77-89.

4 Milne LM, Kyi MS, Chiodini PL, et al. Accuracy of routine laboratory diagnosis of malaria in the United Kingdom. J Clin Pathol 1994;47:740-2.

5 Snell JJS. Problems in susceptibility testings - findings of UK NEQAS for microbiology. J Antimicrob Chemother 1994;33:1-4

\section{$\mathrm{ECHO}$}

\section{Screening for familial Mediterranean fever makes sense in Greece}

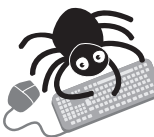

Please visit the Journal of

Clinical

Pathology website [www. jclinpath.com] for a link to the full text of this article.
D eople in Greece may be spared the risk of amyloidosis or unnecessary operations by testing for pyrin gene mutations to diagnose familial Mediterranean fever (FMF) early, according to a molecular study. Further, detailed molecular studies are needed to identify rare or new mutations among the population.

Pyrin mutations were found in 53 of 62 patients of Mediterranean origin diagnosed clinically as having FMF. Forty two were homozygous or compound heterozygous for pyrin mutations; 11 had just one mutation; and nine had no mutations. Among 33 patients with definite FMF, 29 were homozygous or compound heterozygous; three had one mutation; and one none. For 29 patients with probable FMF, 13 were homozygous or compound heterozygous; eight had one mutation, and eight no mutation. Eight different pyrin mutations were found in Greek patients, most commonly E148Q (24\%) and M694V (46\%).

The 62 patients were all referred with definite or probable FMF; 48 were Greek and the others Armenian, Jewish, or Arab. Each was tested for nine of more than 28 known $M E F V$ point mutations.

FMF is an inherited inflammatory condition with a relapsing course that produces fever; synovitis; and abdominal, chest, and skin signs and in the long term may result in amyloidosis. Mediterranean peoples are most at risk. FMF is rarely diagnosed in Greece, but significant migration of other racial groups with a high incidence of the disease into the country suggests that it may become widespread. Therefore reliable screening would be warranted.

A Annals of the Rheumatic Diseases 2003;62:479-481. 